\title{
Oscillatory phenomena in a solar network region
}

\author{
Georgia Tsiropoula ${ }^{1}$, Kostas Tziotziou ${ }^{1}$, Pavol Schwartz ${ }^{2}$ \\ and Petr Heinzel ${ }^{2}$ \\ ${ }^{1}$ Institute for Space Applications and Remote Sensing, National Observatory of Athens, \\ Lofos Koufos, 15236 P. Penteli, Greece \\ email: [georgia; kostas] @space.noa.gr \\ ${ }^{2}$ Academy of Sciences of the Czech Republic, Astronomical Institut, \\ CZ-25165 Ondřejov, Czech Republic \\ email: [schwartz; pheinzel] @asu.cas.cz
}

\begin{abstract}
We examine oscillatory phenomena in a solar network region from multi-wavelength, observations obtained by the ground-based Dutch Open Telescope (DOT), and by instruments on the spacecraft Solar and Heliospheric Observatory (SoHO). The observations were obtained during a coordinated observing campaign on October 14, 2005. The temporal variations of the intensities and velocities in two distinct regions of the quiet Sun were investigated: one containing several dark mottles and the other several bright points defining the network boundaries (NB). The aim is to find similarities and/or differences in the oscillatory phenomena observed in these two regions and in different spectral lines formed from the chromosphere to the transition region, as well as propagation characteristics of waves.
\end{abstract}

Keywords. Sun: chromosphere; transition region; oscillations

\section{Introduction}

In the chromosphere, the quiet Sun displays a distinct network appearance identical to the photospheric supergranular structure. In the $\mathrm{H} \alpha$ line, especially in its wings, several elongated dark structures, called mottles, outline the cell boundaries. In the transition region, the network stands out as more stable cellular patterning with bright patches identifying the network boundaries (NB) and enclosing dark areas which correspond to the internetwork (IN). This spatial dichotomy is also apparent in the power spectra leading to the suggestion that different physical mechanisms may dominate in each region. Several authors have studied properties of spectral lines and reported that the chromospheric plasma localized in NB oscillates with a dominant period of $\sim 300 \mathrm{~s}$ (Lites et al. 1993; Curdt \& Heinzel 1998). Cauzzi et al. (2000) from the power spectrum computed for the intensity of $\mathrm{H} \alpha$ line center found that for both the NB and IN the power distribution peaks at $\sim 300 \mathrm{~s}$ with no enhanced power detectable in the $3 \mathrm{~min}$ range in the IN. This is consistent with observations in the Ca II H line by Lites et al. (1993) showing a power peak in the $3 \mathrm{~min}$ range only for the velocity fluctuations. Tziotziou et al. (2004) from a wavelet analysis of $\mathrm{H} \alpha$ observations found intensity and velocity periodicities in dark mottles outlining the NB, and thus overlying the IN, in the range 270-450 s, although a $180 \mathrm{~s}$ period was also apparent.

In this work we present observations of a solar network region obtained simultaneously by space-borne and ground-based instruments in several spectral lines. We analyze the fluctuations observed through wavelet and global phase difference analyses in order to search for wave signatures at different heights of the solar atmosphere. 

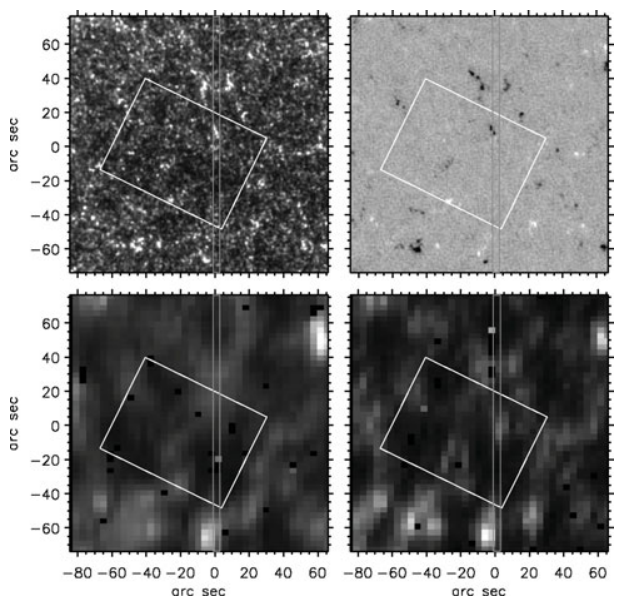

Figure 1. First row: CIV TRACE image (left) and MDI magnetogram (right) obtained at 10:15 UT. Second row: Cut-outs of the CDS raster observations of intensities at He I (left) and O V (right) lines obtained from 10:46 to 11:17 UT and corrected for solar rotation due to the time difference. Solar North is up. The white rectangle inside the images marks the DOT's FOV (pointing to the celestial North), while the two parallel grey lines inside the images mark the location of the sit-and-stare CDS observations.

\section{Observations}

Co-temporal observations of a quiet region found at the solar disk center were obtained on October 14, 2005 by SoHO (CDS and MDI), the Dutch Open Telescope (DOT) and TRACE. DOT obtained a time sequence in $\mathrm{H} \alpha$ which consists of 26 speckle reconstructed images taken simultaneously at a cadence of $35 \mathrm{~s}$ with a pixel size of $0.071^{\prime \prime}$ in 5 wavelengths along the $\mathrm{H} \alpha$ line profile (i.e. at $-0.7 \AA,-0.35 \AA$, line center, $0.35 \AA$ and $0.7 \AA$ ). CDS obtained sit-and-stare observations in several spectral lines among which He I, O v, and Ne VI (effective pixel size of $4 \AA$ in the horizontal and $3.36 \AA$ in the vertical direction) with 60 exposures and cadence of $49 \mathrm{~s}$. CDS raster scans, as well as MDI magnetograms and TRACE filtergrams were used for the co-alignment of the different data sets (Fig. 1). DOT images were rebinned to CDS spatial pixel sizes both in the X-and Y-direction for intercomparison.

\section{Results}

In CDS sit-and-stare observations the quiet Sun is dominated by a pattern of bright streaks defining the NB areas, dark streaks defining the IN areas, while mottles regions appear darker than the NB, but brighter than the IN. In the rebinned DOT $\mathrm{H} \alpha$ line center images a bright patch is observed in the place where network bright points were observed and a dark patch in the respective region of dark mottles. A strong positive correlation exists between respective peak intensities of the CDS lines suggesting a strong association between different heights of the solar atmosphere and indicating that we are possibly observing manifestations of the same structural topology at different heights.

\subsection{Wavelet power analysis}

A wavelet analysis is performed in both intensity and Doppler velocity variations, in two regions (NB and mottles' region), for both unfiltered and filtered with a high-pass frequency filter time series of DOT and CDS observations. $\mathrm{H} \alpha$ line-center intensity variations show a most prominent peak at $\sim 300 \mathrm{~s}$ in the mottles' region and an extended 

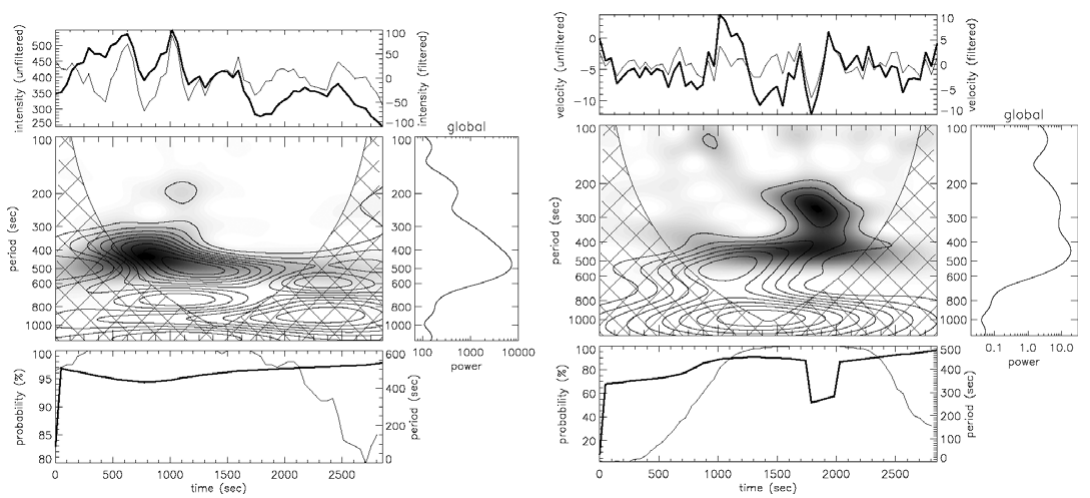

Figure 2. Wavelet analysis at a fixed location of the O v peak intensity (left) and velocity (right) variations in the mottles' region.
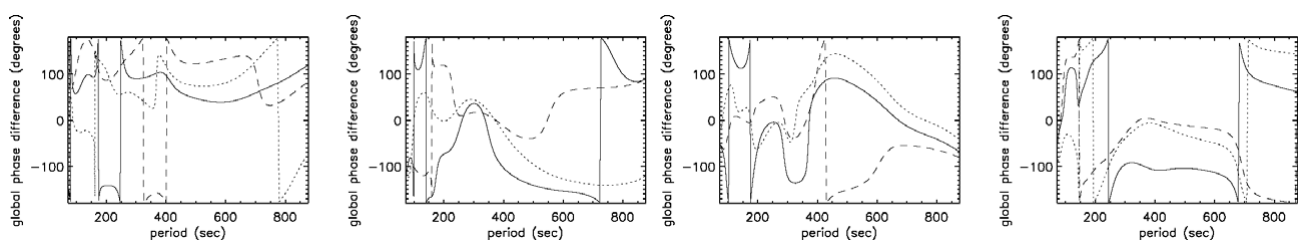

Figure 3. Global phase difference (in degrees) of intensities and velocities as a function of period relative to the $\mathrm{H} \alpha$ line center intensity and Doppler velocity (He I (thick solid line), $\mathrm{OV}$ (dotted line), Ne VI (dashed line)) for the mottles' region (first and third plot, respectively). The same for the NB region (second and fourth plot, respectively).

peak at periods of 300 to $600 \mathrm{~s}$ in the NB region. Unfiltered CDS spectra are dominated by a period $\sim 1000 \mathrm{~s}$, which is subjected to edge effects and has an unclear physical importance. Both CDS intensity and velocity filtered power spectra show several periods with significant power within the cone-of-influence (COI). Most spectra in the mottles' region, as well as in the NB, show dominant oscillation signatures mainly in the $250 \mathrm{~s}$ $400 \mathrm{~s}$ range with rather variable probability (Fig. 2, for details see Tsiropoula et al. 2009).

\subsection{Global phase difference analysis}

Phase difference obtained with a cross-wavelet transform between the $\mathrm{H} \alpha$ line-center filtered intensity and Doppler velocity time series and the corresponding filtered time series of the CDS lines is used for the study of propagation characteristics of waves at different heights in the solar atmosphere (Fig. 3). Phase difference curves have similar behavior indicating a clear interconnection between processes in the lower and higher solar atmosphere and in both mottles and NB regions. Differences in phase difference for the examined spectral lines suggests formation at slightly different heights. I-I (i.e. between intensities) phase spectra depend on many parameters (i.e. frequency, peculiarities of the line formation) and are difficult to interpret. $\mathrm{V}-\mathrm{V}$ (i.e. between velocities) phase differences in the NB show vertically propagating waves, since above $200 \mathrm{~s}$ the inclination of the phase curve with period is constant for all lines as expected from the theoretical behavior of such waves. For periods above $350 \mathrm{~s}$, the phase difference becomes almost constant indicating no propagation, as expected from the NB cut-off period which is about 350-400 s. Non-constant dependence of phase curves on the period above $200 \mathrm{~s}$ in the mottles' region probably indicates: a) non-vertical propagation or b) waves propagating at different inclined mottles along the line-of-sight. The mostly negative phase difference suggests a downward propagation at least for periods of 250-400 s, representing 
waves refracted from the inclined magnetic field of mottles or waves converted to other wave types, such as fast or slow MHD modes (depending on the field strength).

\section{Acknowledgements}

This work was partly supported by a grant PENED 03ED554 [co-financed by ECEuropean Social Fund (80\%) and the Greek Ministry of Development-GSRT (20\%)]. P.S. and P.H. are supported by the ESA-PECS project No 98030. Dr E. Khomenko is thanked for very useful discussions concerning the intrepretation of phase differences and waves. The authors are also grateful to the DOT, SoHO and TRACE observers and science planning teams.

\section{References}

Cauzzi, G., Falchi, A., \& Falciani, R. 2000, A\&A, 357, 1093

Curdt, W. \& Heinzel, P. 1998, ApJ(Letters), 503, L95

Lites, B. W., Rutten, R. J., \& Kalkofen, W. 1993, ApJ, 414, 345

Tziotziou, K., Tsiropoula, G., \& Mein, P. 2004, A\&A, 423, 1133

Tsiropoula, G., Tziotziou, K., Schwartz, P., \& Heinzel, P. 2009, A\&A, 493, 217 